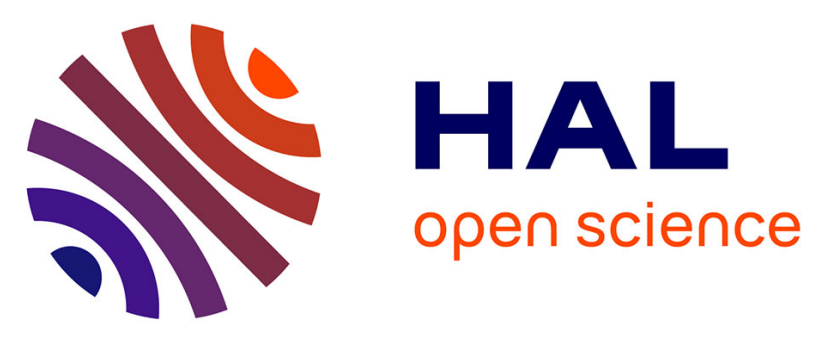

\title{
Broadening of cohesinopathies: Exome sequencing identifies mutations in ANKRD11 in two patients with Cornelia de Lange-overlapping phenotype
}

Ilaria Parenti, Cristina Gervasini, Jelena Pozojevic, Luitgard Graul-Neumann, Jacopo Azzollini, Diana Braunholz, Erwan Watrin, Kerstin S. Wendt, Anna Cereda, Davide Cittaro, et al.

\section{To cite this version:}

Ilaria Parenti, Cristina Gervasini, Jelena Pozojevic, Luitgard Graul-Neumann, Jacopo Azzollini, et al. Broadening of cohesinopathies: Exome sequencing identifies mutations in ANKRD11 in two patients with Cornelia de Lange-overlapping phenotype. Clinical Epigenetics, 2016, 89 (1), pp.74-81. 10.1111/cge.12564 . hal-01117251

HAL Id: hal-01117251

https://hal-univ-rennes1.archives-ouvertes.fr/hal-01117251

Submitted on 6 May 2015

HAL is a multi-disciplinary open access archive for the deposit and dissemination of scientific research documents, whether they are published or not. The documents may come from teaching and research institutions in France or abroad, or from public or private research centers.
L'archive ouverte pluridisciplinaire HAL, est destinée au dépôt et à la diffusion de documents scientifiques de niveau recherche, publiés ou non, émanant des établissements d'enseignement et de recherche français ou étrangers, des laboratoires publics ou privés. 


\section{Broadening of cohesinopathies: Exome sequencing identifies mutations in $A N K R D 11$ in two patients with Cornelia de Lange-overlapping phenotype}

Ilaria Parenti ${ }^{1,2}$, Cristina Gervasini ${ }^{1}$, Jelena Pozojevic ${ }^{2}$, Luitgard Graul-Neumann ${ }^{3}$, Jacopo Azzollini ${ }^{1}$, Diana Braunholz ${ }^{2}$, Erwan Watrin ${ }^{4}$, Kerstin S. Wendt ${ }^{5}$, Anna Cereda ${ }^{6}$, Davide Cittaro ${ }^{7}$, Gabriele Gillessen-Kaesbach ${ }^{8}$, Dejan Lazarevic $^{7}$, Milena Mariani ${ }^{6}$, Silvia Russo ${ }^{9}$, Ralf Werner $^{10}$, Peter Krawitz ${ }^{3,11}$, Lidia Larizza ${ }^{1,9}$, Angelo Selicorni $^{6, *}$, Frank J. Kaiser ${ }^{2, *}$,

*share last authorship

\#corresponding author

${ }^{1}$ Medical Genetics, Department of Health Sciences, Università degli Studi di Milano, Milan, Italy

${ }^{2}$ Sektion für Funktionelle Genetik am Institut für Humangenetik Lübeck, Universität zu Lübeck, Germany

${ }^{3}$ Ambulantes Gesundheitszentrum der Charité Campus Virchow, Humangenetik, Universitätsmedizin Berlin, Berlin, Germany

${ }^{4}$ Institut de Génétique et Développement de Rennes, UMR6290-CNRS, Faculté de Médecine, Rennes, France

${ }^{5}$ Department of Cell Biology, Erasmus MC, Rotterdam, Netherlands

${ }^{6}$ U.O.S. Genetica Clinica Pediatrica, Clinica Pediatrica Fondazione MBBM, A.O. S.Gerardo, Monza, Italy

${ }^{7}$ Centre for Translational Genomics and Bioinformatics, San Raffaele Scientific Institute, Milan, Italy

${ }^{8}$ Institut für Humangenetik Lübeck, Universität zu Lübeck, Germany

${ }^{9}$ Laboratory of Medical Cytogenetics and Molecular Genetics; IRCCS Istituto Auxologico Italiano; Milan, Italy

${ }^{10}$ Department of Paediatrics and Adolescent Medicine, Division of Experimental Paediatric Endocrinology and Diabetes, University of Lübeck, Lübeck, Germany

${ }^{11}$ Max Planck Institute for Molecular Genetics, Berlin, Germany

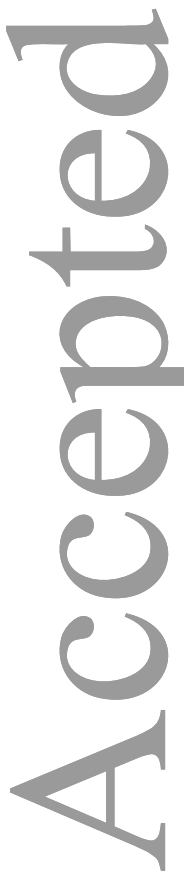

Corresponding author:

Frank Kaiser

Frank.Kaiser@uksh.de

Universität zu Lübeck

Sektion für Funktionelle Genetik

am Institut für Humangenetik

Ratzeburger Allee 160

23538 Lübeck

Tel.: +494515002623

Fax: +494515004861

Conflict of interest statement: The authors declare no conflict of interest.

This article has been accepted for publication and undergone full peer review but has not been through the copyediting, typesetting, pagination and proofreading process, which may lead to differences between this version and the Version of Record. Please cite this article as doi: 10.1111/cge.12564 


\section{Acknowledgements}

We thank Mariani Foundation, Milan, for the support of the clinical activity of UOS Pediatric Genetic Clinic of MBBM Foundation Monza. The study was supported under the frame of ERare-2 (TARGET-CdLS) by the German Federal Ministry of Education and Research (BMBF) (to FJK), by the by the Netherlands Organization for Health Research and Development (ZonMW) (to KSW), by the Agence Nationale de la Recherche (ANR) (to EW) and the "Schwerpunktprogramm (SPP) für medizinische Genetik" of the University of Lübeck (to DB, RW and FJK). 


\begin{abstract}
Cornelia de Lange syndrome (CdLS) and KBG syndrome are two distinct developmental pathologies sharing common features such as intellectual disability, psychomotor delay, and some craniofacial and limb abnormalities. Mutations in one of the five genes NIPBL, SMC1A, SMC3, HDAC8 or RAD21, were identified in at least $70 \%$ of the patients with CdLS. Consequently, additional causative genes, either unknown or responsible of partially merging entities, possibly account for the remaining $30 \%$ of the patients. In contrast, KBG has only been associated with mutations in ANKRD11.

By exome sequencing we could identify heterozygous loss-of-function mutations in ANKRD11 in two patients with the clinical diagnosis of CdLS. Both patients show features reminiscent of CdLS such as characteristic facies as well as a small head circumference which is not described for KBG syndrome. Patient A, who carries the mutation in a mosaic state, is a four-year-old girl with features reminiscent of CdLS. Patient B, a 15-year-old boy, shows a complex phenotype which resembled CdLS during infancy, but has developed to a more KBG overlapping phenotype during childhood. These findings point out the importance of screening ANKRD11 in young CdLS patients who were found to be negative for mutations in the five known CdLS genes.
\end{abstract}

Key words: ANKRD11, cohesin, Cornelia de Lange syndrome, KBG syndrome, mosaicism, whole exome sequencing

\title{
Introduction
}

Cornelia de Lange syndrome (CdLS, OMIM \#122470, 300590, 610759, 300882, and 614701) and KBG syndrome (OMIM \#148050) are two distinct rare multisystem developmental disorders. 
CdLS is characterized by clinical and genetic heterogeneity (1). The main features of the syndrome are pre- and post-natal growth retardation, intellectual disability, psychomotor delay, limb anomalies and a typical facies. These distinctive facial features include a low anterior hairline, arched eyebrows, synophrys, depressed nasal bridge, anteverted nares, long and smooth philtrum and thin upper lip with down-turned corners of the mouth. The cardiac, gastro-intestinal and genitourinary systems are also frequently involved (1). Heterozygous mutations in five different genes have been identified in patients with CdLS: NIPBL (5p13.2, OMIM \#608667), SMC1A (Xp11.22, \#300040), SMC3 (10q25.2, \#606062), HDAC8 (Xq13.1, \#300269) and RAD21 (8q24.11, \#606462). All five genes are associated with the cohesin complex and its regulation. While the proteins SMC1A, SMC3 and RAD21 represent structural components of the cohesin complex, NIPBL and HDAC8 are associated regulators: NIPBL mediates the cohesin loading onto DNA and HDAC8 promotes SMC3 deacetylation to regulate proper cohesin dissociation from chromatin (2-7). The cohesin complex is involved in a large spectrum of functions including sister chromatid cohesion, DNA damage response, chromatin modification, transcriptional regualtion and long-range interactions between distant genomic regions (8).

NIPBL is the major gene of the syndrome and alterations can be identified in more than half of the patients (9). Mutations in NIPBL are usually associated with a severe phenotype and with a high frequency of limb anomalies (9). Recently, a high proportion of mosaic mutations have been identified in patients with CdLS, thus partially accounting for the wide variability of expression: more than $20 \%$ of patients who were mutation negative in peripheral blood have been shown to carry mutations in NIPBL on DNA extracted from buccal mucosa or fibroblasts $(10,11)$. 
Besides NIPBL, the remaining four genes account for about $15 \%$ of the patients, who are usually characterized by a more moderate or milder phenotype as those with mutations in NIPBL $(6,7,12,13)$.

KBG syndrome is a very rare genetic disorder characterized by macrodontia of the upper central incisors, short stature, skeletal anomalies, psychomotor delay, intellectual disability, seizures, and craniofacial abnormalities which include a round facies, brachycephaly, hypertelorism, a bulbous nasal tip, long philtrum and broad and arched eyebrows $(14,15)$. KBG has been associated with loss of function mutations in the ANKRD11 gene (16q24.3, \#611192), which codes for the ankyrin repeat-containing protein 11, also known as ankyrin repeat-containing cofactor 1 (ANCO-1) (16). This protein is known to inhibit the transcriptional activation of nuclear receptors target genes through the recruitment of the histone deacetylases HDAC3, HDAC4 and HDAC5 (17). Sirmaci et al. (16) demonstrated that ANKRD11 localizes within the nuclei of neurons and accumulates in discrete inclusions when neurons are depolarized, suggesting a role in neural plasticity. The protein contains five ankyrin repeats involved in protein-protein interactions, two repression domains (at the Nand C-terminus) and one activation domain capable of stimulating transcription (18).

Herein we report on two patients with a tentative clinical diagnosis of CdLS who were found to be negative for mutations in the five known CdLS genes but carry heterozygous loss-offunction mutations in ANKRD11 identified by exome-sequencing approaches.

\section{Patients and methods}

\section{$\underline{\text { Patients }}$}

Patient A (Fig. 1 a-h) is a German female, the fourth offspring of healthy and nonconsanguineous parents. The patient was born at 37 weeks of gestation after caesarian section. The Apgar scores were 9 and 10 at 1 and 5 minutes, respectively. Birth weight was 
2350 g (-1.6 SD), birth length was 46 cm (1.75 SD) and the Occipital Frontal Circumference (OFC) was $31.5 \mathrm{~cm}$ (- $2 \mathrm{SD}$ ). The patient showed early feeding difficulties resulting in a poor increase of weight. She also showed general muscular hypertonia.

The patient was re-evaluated at the age of four months. At this age, weight was $5600 \mathrm{~g}(+0.3$ SD), height was $59 \mathrm{~cm}(-1.75 \mathrm{SD})$ and OFC was $38.3 \mathrm{~cm}(-0.3 \mathrm{SD})$. She showed a small brachycephalic head, open anterior fontanelle, synophrys, downslanting palpebral fissures, anteverted nares, depressed nasal bridge, long philtrum, thin lips with downturned corners of the mouth, short neck, mild brachydactyly, proximally set thumbs and mild micrognathia. She presented with impressive hypertonia. She was able to sit at the age of 16 months, and to walk without support at the age of 20 months.

Last evaluation was performed at the age of four years. At this age, the patient was not able to eat without assistance. She presented with speech delay, being able to speak about 50 words and showed an aggressive and self-injurious behavior associated with autism. She showed a moderate intellectual disability. Her gait was wide based. Hearing or vision impairment were not observed.

Based on these clinical features the patient was considered to have CdLS.

Patient B (Fig. $1 \mathrm{i}-\mathrm{k}$ ) is an Italian male and the third child of healthy and non-consanguineous parents. Birth weight was 3150 g (-1.7 SD), length was $53 \mathrm{~cm}$ (0.3 SD) and the OFC was 33 cm (-2.9 SD). A bilateral vesico-ureteral reflux was surgically treated in the first year of life. Moreover, ultrasound kidney evaluation showed kidney dysplasia. Subsequent analysis of the renal function indicated the presence of a mild non nephrotic proteinuria without impairment of kidney function. He presented gastro-esophageal reflux with esophagitis of first degree. For this reason, he received proton pump inhibitors therapy.

At the age of four he presented with moderate psychomotor and cognitive delay. He was able to walk independently, to speak in sentences and was completely able to manage himself in 
daily life. At the age of nine he started to show severe behavioral problems, characterized by auto- and hetero-aggression. He also showed generalized epilepsy.

At the age of 15 years the boy was in a good health condition. His weight was $41.2 \mathrm{~kg}(-0.5$ SD), height was $161 \mathrm{~cm}(-1.6 \mathrm{SD})$ and the OFC was $54 \mathrm{~cm}(-1.2 \mathrm{SD})$.

He showed the following facial dysmorphisms: Low anterior hairline, broad and arched eyebrows with synophrys, long eyelashes, high nasal bridge, large nasal tip, smooth philtrum, large mouth with thin lips, macrodontia of superior central incisors, mild micrognathia. He also showed mild lumbar and forearms hirsutism, myopia and astigmatism, proximally placed thumbs and clinodactyly of the fifth finger. He was clinically diagnosed as having CdLS during childhood.

Methods

DNA extraction and sequencing

Genomic DNA was isolated from peripheral blood, buccal mucosa and fibroblasts. Extraction from blood and fibroblasts was performed with the QIAamp DNA Mini Kit (Qiagen, Milan, Italy), while the PureGene Buccal Cell Core Kit A (Qiagen) was used for gDNA isolation from buccal mucosa cells. PCR products were sequenced using the Big Dye terminator v3.1 Sequencing Kit and run on the ABI PRISM 3130xl sequencer (Applied Biosystems, Foster City, CA, USA). Electropherograms were analyzed with ChromasPro Software version 1.7.6 (Technelysium Pty Ltd, Tewantin QLD, Australia) and aligned with the wild type ANKRD11 sequence (RefSeq NM_013275).

Gene panel

Targeted gene panel analysis was performed on the fibroblast DNA of both patients with the Personal Genome Machine (Ion Torrent PGM, Life Technologies, Darmstadt, Germany) as described by Braunholz et al. (11).

Exome sequencing 
Targeted enrichment was performed via Sure Select All Exon Kit V2 (Agilent, Waldbronn, Germany) for patient A, and through the TruSeq Exome Enrichment preparation kit (Illumina) for patient B. Sequencing was performed with Illumina’s HiSeq 2500 system.

For bioinformatics analysis, read tags were aligned on human reference genome (GRCh37.5) using bwa 0.6.1 (19) or Novoalign (Novocraft Technologies, Selangor, Malesia). Single nucleotide variants (SNVs) and short insertions and deletions (indels) were called with GATK according to the best practice guidelines. The variant annotation on a functional level was performed using Jannovar or snpEff (20) and filtered in GeneTalk for somatic variants (21).

\section{Pyrosequencing}

The allelic dosage was quantified with an assay specific for each patient. The analysis was performed with the Pyro Gold Reagent Kit on the Pyro MarkID Q24 (Qiagen).

\section{Results}

Initial Sanger sequencing as well as high coverage target-enriched gene panel sequencing could not detect any pathogenic mutation in the CdLS-associated genes in either of the patients. Because of the high frequency of mosaic mutations in patients with CdLS that cannot be detected in DNA derived from blood, buccal mucosa and fibroblasts were also analyzed, as suggested by Braunholz et al. (11).

Since no obvious disease-causing mutation could be identified in any of the DNA samples, both patients were selected for a whole exome-sequencing analysis, which was performed on blood DNA samples.

For patient A four possible de-novo variants were considered as valid candidates: one splicing mutation in the Bone Morphogenetic Protein Receptor 2 (BMPR2) gene, two missense mutations in the ACAP3 (ArfGAP with Coiled-coil, Ankyrin repeat and PH 
domains 3) and in the ERC2 genes (ELKS/RAB6-interacting/CAST family member 2) as well as a nonsense mutation in ANKRD11. No obvious functional correlation was found between the first three genes and the cohesin complex. Moreover, three different bioinformatic tools (PolyPhen-2, SIFT and Mutation Taster) predicted the first three variants to be not pathogenic. Besides, mutations in $A N K R D 11$ were recently identified in three patients with CdLS-overlapping phenotypes (22). The ANKRD11 mutation of patient A, c.5483G>T; p.S1828X, was found in 31\% (836) of the 2664 sequencing reads, indicating a possible mosaic state.

For patient B four variants were detected. Three genes, namely ZW10 (Centromere/Kinetocore protein zw10 homolog), SMC1B (Structural Maintenance of Chromosome protein 1B) and ASPM (Abnormal Spindle-like Microcephaly-associated Protein), carried a missense mutation, while a four base-pairs deletion resulting in a frameshift was identified in the $A N K R D 11$ gene. All three missense variants were found to be inherited; therefore, we focused on the frame-shift deletion affecting exon 9 of ANKRD11 (c.2297_2300delAGAA, p.K766_K767fsX9). Similar to the analysis of patient A, an uneven distribution of the wild type to the mutant allele (47/13 reads) was detected, thus raising the suspicion of a mosaicism.

Both ANKRD11 mutations were confirmed and proven as de novo by Sanger sequencing on different tissues (Fig. 2a). While the electropherograms of patient A further indicated an unequal ratio between the wild type and the mutant allele, no significant difference in the distribution of the two alleles could be observed in patient B. To further investigate the role of the putative mosaicism for both mutations we performed pyrosequencing analyses. By this, we could confirm the presence of a mosaic state for patient A while the mutation in patient B was excluded to be mosaic. In detail, the quantification of the two alleles in patient A showed a 30:70 ratio between the mutant and the wild type allele in blood DNA and a 50:50 ratio on 
fibroblasts DNA, whereas equal distribution of wild type and mutant allele was observed in blood, buccal mucosa as well as fibroblast DNA of patient B (Fig. 2b).

\section{Discussion}

Although CdLS and KBG are rare distinct syndromes, both show overlapping features such as cognitive impairment, growth retardation and some craniofacial abnormalities including brachycephaly, broad arched eyebrows and anteverted nostrils. Limb anomalies, specifically small hands and feet, clinodactyly of the fifth finger and syndactyly of the second and third toes, are also described in both syndromes. In this context, it is worth to be noted that both ANKRD11 and the cohesin complex are involved in gene expression regulation. The main function of ANKRD11 is to repress the transcriptional activation of target genes of nuclear receptors by recruiting deacetylases to different promoters (17). Similarly, the cohesin complex is involved in the regulation of gene expression, mediating both transcriptional activation and repression $(23,24)$. It is therefore tempting to speculate that the dysregulation of functionally interconnected sets of genes due to deficiency of the cohesin complex or ANKRD11 might result in overlapping phenotypical features.

Despite some shared phenotypical features, both syndromes must be considered as two distinct entities. In this regard, the head circumference and the teeth conformation may be used as main distinguishing features: CdLS patients are normally characterized by a marked microcephaly, which is not described for patients with KBG. Teeth anomalies including macrodontia of the central incisors, fused incisors, malposition, oligodontia and overcrowded teeth are instead frequent in patients with KBG, and not typical for CdLS (Table 1).

Here we report on two CdLS patients with a mutation in ANKRD11. Patient A shows some clinical signs common to both CdLS and KBG, specifically brachycephaly, short neck, arched eyebrows, anteverted nares, a long philtrum, intellectual disability and behavioral 
problems. Nevertheless, the small head circumference, synophrys, long eyelashes, a depressed nasal bridge and the absence of macrodontia or fused incisors were suggestive for the clinical diagnosis of CdLS. Patient B also shows features present in CdLS and KBG, such as broad and arched eyebrows, long philtrum, clinodactyly, intellectual disability and behavioral problems. Typical for CdLS are the low anterior hairline, synophrys, long eyelashes, smooth philtrum, thin lips with downturned corners of the mouth and a small head circumference. Despite the presence of a fifth finger clinodactyly, the hands of patient B show long fingers which is rather atypical for CdLS (Fig. 1k). Suggestive for KBG are the large nasal tip and macrodontia of the central upper incisors. Although patient B showed typical clinical features reminiscent for CdLS during early childhood, at the age of 15 years his phenotype was more compatible with KGB.

Interestingly, patient A carries the mutation in a mosaic state. In the last years, mosaicism has been proved to play an important role in CdLS (10).

So far, only one mosaic mutation in the ANKRD11 gene has been reported in the mildly affected mother of a KBG proband (25). In our patient A it might be possible that the presence of mosaicism with a variable ratio of the wild type and mutant gene product in different tissues can contribute to the mitigation of the KBG features and may explain the more CdLS-overlapping phenotype.

In summary, mutations in $A N K R D 11$ can result in a CdLS-overlapping phenotype which seems to be most distinct in early childhood. Therefore, a detailed clinical follow up is strongly recommended. Sequencing analysis of ANKRD11 has to be considered for those patients with the suspected clinical diagnoses of CdLS during childhood who were tested as mutation negative in the five known CdLS genes. 


\section{References}

1. Kline AD, Krantz ID, Sommer A et al. Cornelia de Lange syndrome: clinical review, diagnostic and scoring systems, and anticipatory guidance. Am J Med Genet A 2007: 143A(12):1287-96

2. Tonkin ET, Wang TJ, Lisgo S, Bamshad MJ, Strachan T. NIPBL, encoding a homolog of fungal Scc2-type sister chromatid cohesion proteins and fly Nipped-B, is mutated in Cornelia de Lange syndrome. Nat Genet 2004: 36(6):636-41

3. Krantz ID, McCallum J, DeScipio C et al. Cornelia de Lange syndrome is caused by mutations in NIPBL, the human homolog of Drosophila melanogaster Nipped-B. Nat Genet 2004: 36(6):631-5

4. Musio A, Selicorni A, Focarelli ML et al. X-linked Cornelia de Lange syndrome owing to SMC1L1 mutations. Nat Genet 2006: 38(5):528-30

5. Deardorff MA, Kaur M, Yaeger D et al. Mutations in cohesin complex members SMC3 and SMC1A cause a mild variant of Cornelia de Lange syndrome with predominant mental retardation. Am J Hum Genet 2007: 80(3):485-94

6. Deardorff MA, Wilde JJ, Albrecht M et al. RAD21 Mutations Cause a Human Cohesinopathy. Am J Hum Genet 2012a: 90(6):1014-27

7. Deardorff MA, Bando M, Nakato R et al. HDAC8 mutations in Cornelia de Lange syndrome affect the cohesin acetylation cycle. Nature 2012b: 489(7415):313-7

8. Mehta GD, Kumar R, Srivastava S, Ghosh SK. Cohesin: functions beyond sister chromatid cohesion. FEBS Lett 2013: 587(15):2299-312

9. Selicorni A, Russo S, Gervasini C et al. Clinical score of 62 Italian patients with Cornelia de Lange syndrome and correlations with the presence and type of NIPBL mutation. Clin Genet 2007: 72(2):98-108 
10. Huisman SA, Redeker EJ, Maas SM, Mannens MM, Hennekam RC. High rate of mosaicism in individuals with Cornelia de Lange syndrome. J Med Genet 2013: 50(5):339-44

11. Braunholz D, Obieglo C, Parenti I et al. Hidden Mutations in CdLS - Limitations of Sanger Sequencing in Molecular Diagnostics. Hum Mutat 2014 Sep 5. doi: 10.1002/humu.22685

12. Gervasini C, Russo S, Cereda A et al. Cornelia de Lange individuals with new and recurrent SMC1A mutations enhance delineation of mutation repertoire and phenotypic spectrum. Am J Med Genet A 2013: 161(11):2909-19

13. Kaiser FJ, Ansari M, Braunholz D et al. Loss-of-function HDAC8 mutations cause a phenotypic spectrum of Cornelia de Lange syndrome-like features, ocular hypertelorism, large fontanelle and X-linked inheritance. Hum Mol Genet 2014: 23(11):2888-900

14. Herrmann J, Pallister PD, Tiddy W, Opitz JM. The KBG syndrome-a syndrome of short stature, characteristic facies, mental retardation, macrodontia, and skeletal anomalies. Birth Defects Orig Artic Ser 1975: 11, 7-18

15. Ockeloen C, Willemsen MH, de Munnik S et al. Further delineation of the KBG syndrome phenotype caused by ANKRD11 aberrations. Eur J Hum Gen 2014. doi: 10.1038/ejhg.2014.253

16. Sirmaci A, Spiliopoulos M, Brancati $\mathrm{F}$ et al. Mutations in ANKRD11 cause KBG syndrome, characterized by intellectual disability, skeletal malformations, and macrodontia. Am J Hum Genet 2011: 89(2):289-94

17. Zhang A, Yeung PL, Li CW et al. Identification of a novel family of ankyrin repeats containing cofactors for p160 nuclear receptor coactivators. J Biol Chem 2004: 279, 33799-33805 
18. Zhang A, Li CW, Chen JD. Characterization of transcriptional regulatory domains of ankyrin repeat cofactor 1. Biochem Biophys Res Commun 2007: 358, 1034-1040

19. Li H and Durbin R. Fast and accurate long-read alignment with Burrows-Wheeler transform. Bioinformatics 2010: 26:589-595

20. Jäger M, Wang K, Bauer S, Smedley D, Krawitz P, Robinson PN. Jannovar: a java library for exome annotation. Hum Mutat. 2014: 35(5):548-55

21. Kamphans T, Krawitz PM. GeneTalk: an expert exchange platform for assessing rare sequence variants in personal genomes. Bioinformatics 2012: 28(19):2515-6

22. Ansari M, Poke G, Ferry Q et al. Genetic heterogeneity in Cornelia de Lange syndrome (CdLS) and CdLS-like phenotypes with observed and predicted levels of mosaicism. J Med Genet 2014: 51(10):659-68

23. Horsfield JA, Anagnostou SH, Hu JK et al. Cohesin-dependent regulation of Runx genes. Development 2007: 134(14):2639-49

24. Wendt KS, Yoshida K, Itoh T et al. Cohesin mediates transcriptional insulation by CCCTC-binding factor. Nature 2008: 451(7180):796-801

25. Khalifa M1, Stein J, Grau L et al. Partial deletion of ANKRD11 results in the KBG phenotype distinct from the 16q24.3 microdeletion syndrome. Am J Med Genet A 2013: 161A(4):835-40 


\section{Legends}

Fig. 1

Phenotype of the patients. (a-h) Patient A: $(a, b)$ frontal and lateral view of the patient at the age of four months. (c) Facial gestalt at the age of four years. (d) Appearance of the teeth. (e) Picture of the left foot. (f-h) Pictures of the left hand, revealing shortened first metacarpal, clinodactily of the fifth finger and proximally set thumb. (i-k) Patient B: (i, j) Facial appearance at the age of eight and 15 years, respectively. (k) Picture of the left hand, showing clinodactyly of the fifth finger and proximally set thumb.

Fig. 2

Analysis of multiple tissues of both patients through Sanger sequencing (a) and pyrosequencing (b).

Sanger sequencing shows an unequal distribution between the mutant and the wild type allele in patient $\mathrm{A}$, but not in patient $\mathrm{B}$. The pyrosequencing analysis confirms the Sanger sequencing results, showing a 50:50 ratio between the two alleles in patient $\mathrm{B}$ and in the fibroblast DNA of patient A; blood DNA of patient A reveals the presence of 32\% of mutant allele, and $68 \%$ of wild type allele, thus confirming the presence of mosaicism. 
Table 1. Overlapping CdLS and KBG clinical features of the reported patients

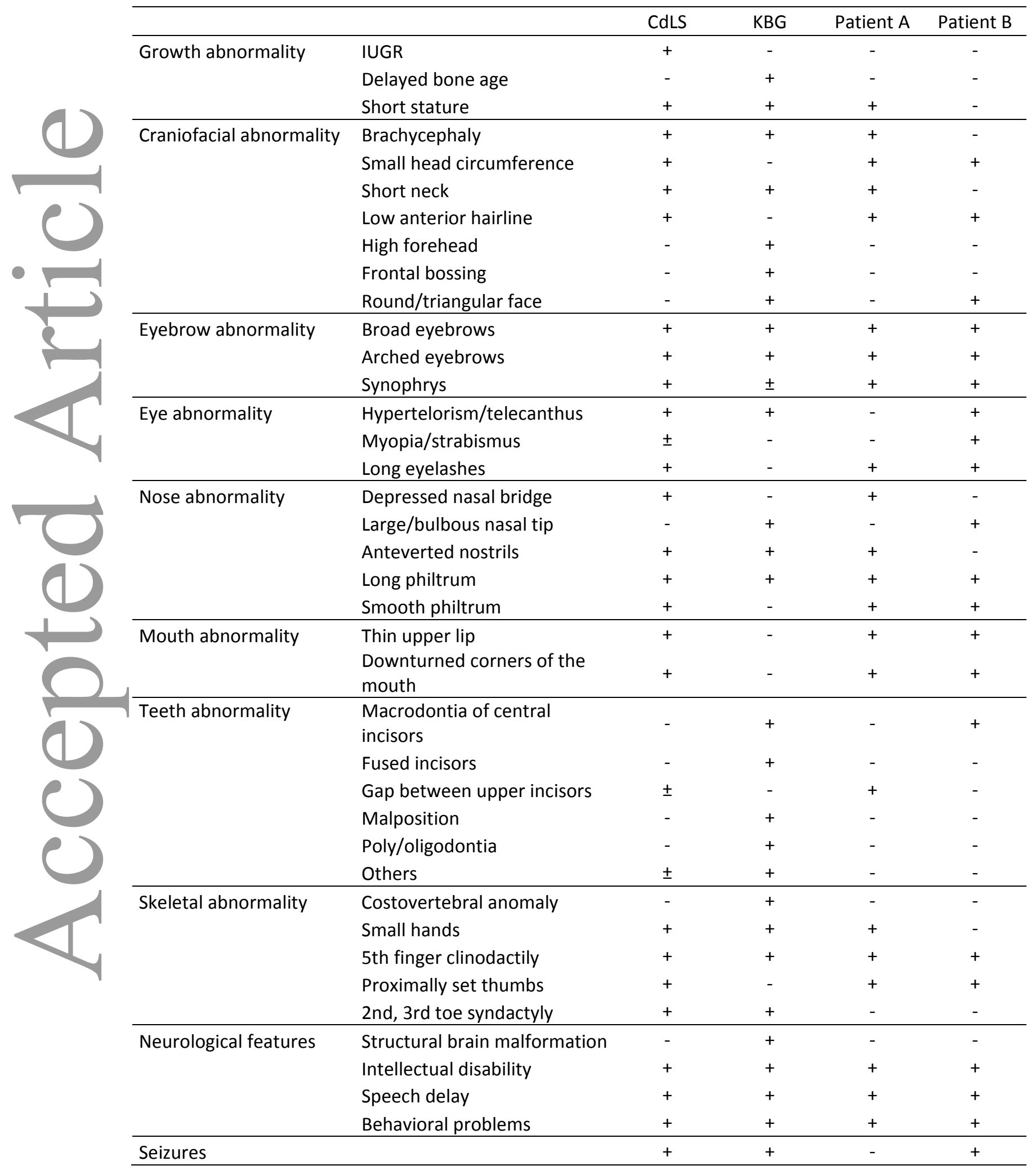




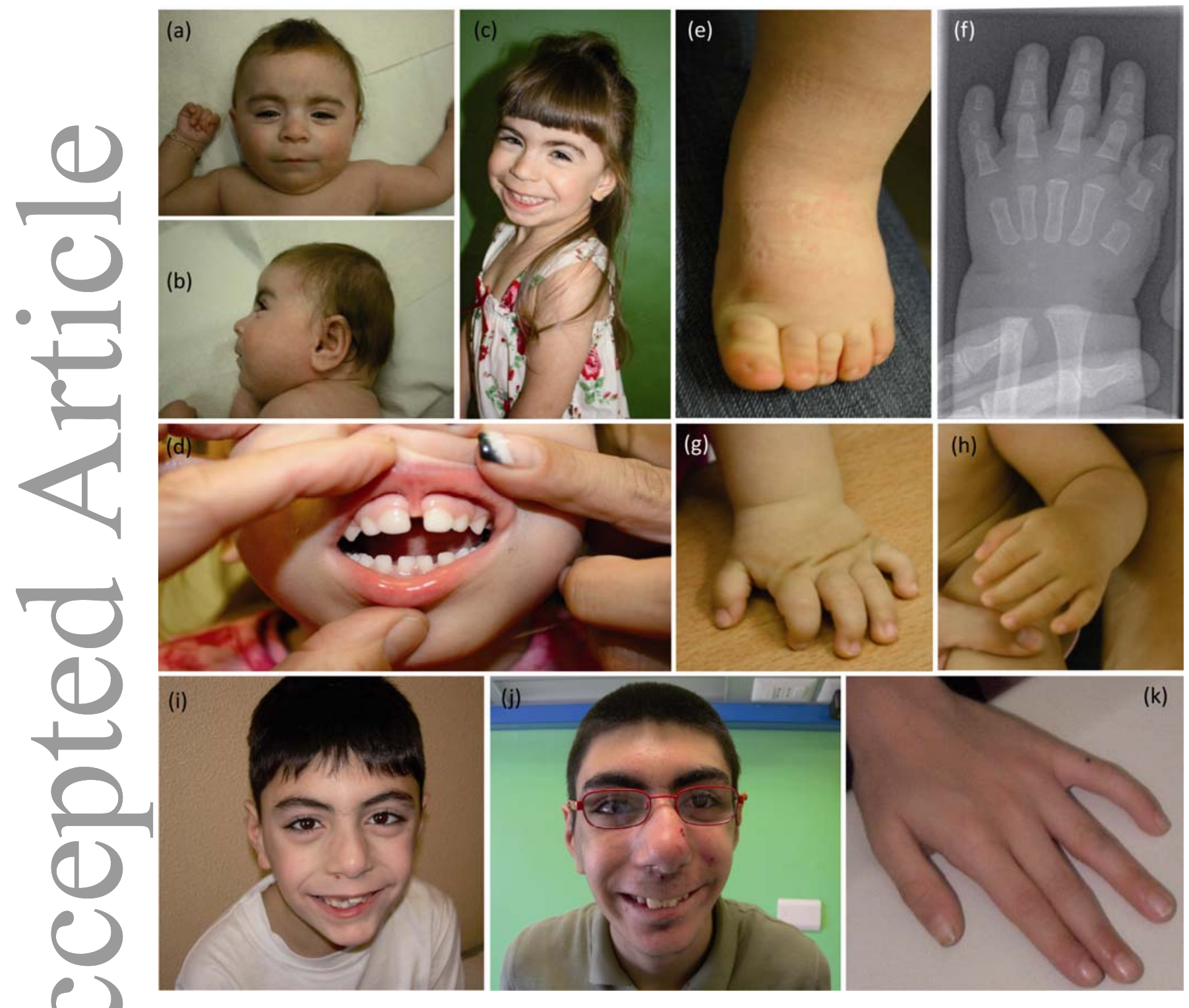

Fig. 1

Phenotype of the patients. (a-h) Patient A: (a, b) frontal and lateral view of the patient at the age of four months. (c) Facial gestalt at the age of four years. (d) Appearance of the teeth. (e) Picture of the left foot. (f-h) Pictures of the left hand, revealing shortened first metacarpal, clinodactily of the fifth finger and proximally set thumb. (i-k) Patient B: (i, j) Facial appearance at the age of eight and 15 years, respectively. (k) Picture of the left hand, showing clinodactyly of the fifth finger and proximally set thumb. 


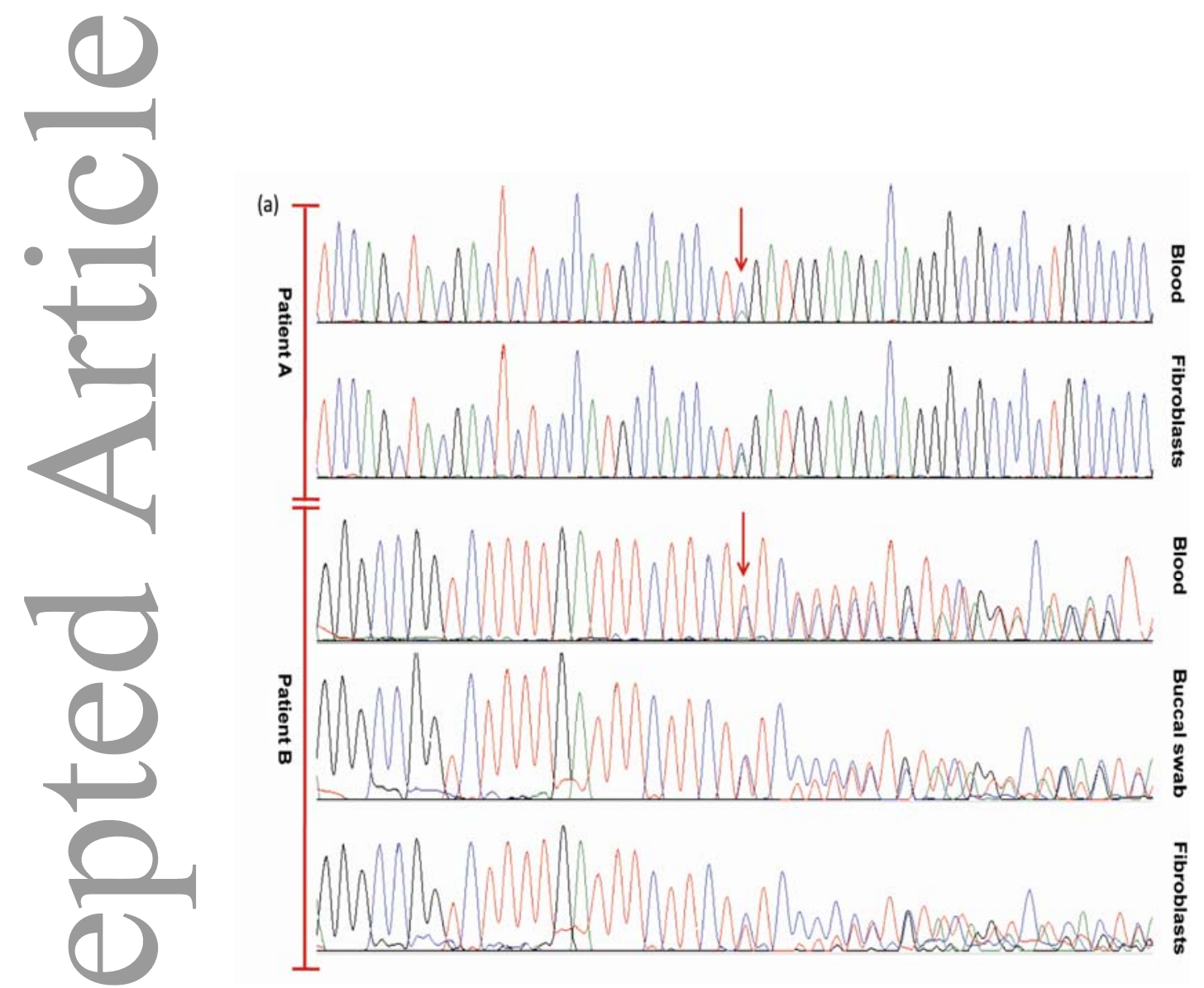




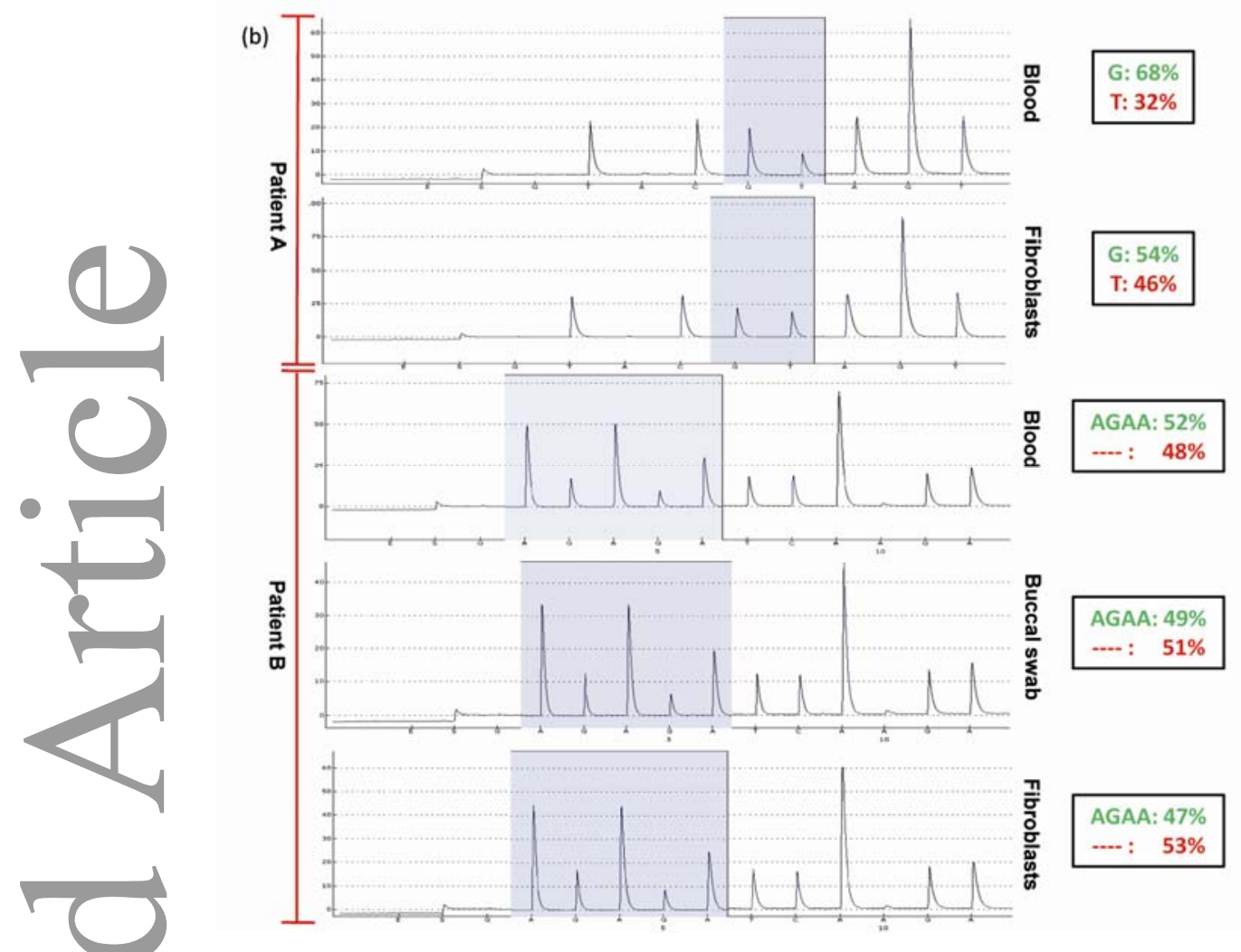

Fig. 2

Analysis of multiple tissues of both patients through Sanger sequencing (a) and pyrosequencing (b).

Sanger sequencing shows an unequal distribution between the mutant and the wild type allele in patient $\mathrm{A}$, but not in patient $\mathrm{B}$. The pyrosequencing analysis confirms the Sanger sequencing results, showing a 50:50 ratio between the two alleles in patient $\mathrm{B}$ and in the fibroblast DNA of patient A; blood DNA of patient A reveals the presence of 32\% of mutant allele, and $68 \%$ of wild type allele, thus confirming the presence of mosaicism. 